\title{
落合芳博*1 \\ Meshless Thermo-Elastoplastic Analysis by Triple-Reciprocity BEM
}

\author{
Yoshihiro OCHIAI*2 \\ *2 Department of Mechanical Engineering, Kinki University, \\ 3-4-1 Kowakae, Higashi-Osaka-shi, Osaka, 577-8502 Japan
}

\begin{abstract}
In general, internal cells are required to solve thermo-elastoplastic problems using a conventional boundary element method (BEM). However, in this case, the merit of BEM, which is the easy preparation of data, is lost. The conventional multiple-reciprocity boundary element method (MRBEM) cannot be used to solve the elastoplasticity problems, because the distribution of initial strain or initial stress cannot be determined analytically. In this paper, it is shown that twodimensional thermo elastoplasticity problems can be solved without the use of internal cells, by using the triple-reciprocity boundary element method and a thin plate spline. Initial strain and initial stress formulations are adopted and the initial strain distribution is interpolated using boundary integral equations and a thin plate spline. A new computer program was developed and applied to several problems.
\end{abstract}

Key Words: Boundary Element Method, Elastoplasticity, Thermal Stress, Computational Mechanics, Initial Strain

\section{1. 緒言}

従来の境界要素法により熱弾塑性解析をする場合, 内部セルが必要になる.内部セルを使用する方法で は, データが容易に作成できるという境界要素法の利 点が損なわれる. また, 従来の境界要素法では熱弾塑 性状態の内部応力を計算する際, 強い特異性の問題を 考慮しなければならなかった. 著者は弾塑性解析にお いても, 三重相反境界要素法によって, 内部セルを用 いないで解析が可能であることを示している.すでに 示した方法では, 熱応力は考慮されていなかった. そ こで, 本論文では, メッシュレスで定常熱応力を考慮 した熱弾塑性解析を行うための解法を, 三重相反境界 要素法を用いて示す. 定式化に際して, 初期ひずみ法 および初期応力法で示す. 初期応力法は初期ひずみ法 とほとんど同じ手法を用いるので, 初期ひずみを中心 に示す.なお，三重相反法で使用する高次基本解は可 能な限り一般解で示した.

\section{2. 理 論}

\section{$2 \cdot 1$ 定常熱伝導解析 境界要素法によれば, 定} 常熱伝導問題を解くための境界積分方程式は次式で与 えられる(1).

$$
\begin{gathered}
C T(P)=\int_{\Gamma}\left[T^{[1]}(P, Q) \frac{\partial T(Q)}{\partial n}\right. \\
\left.-\frac{\partial T^{[1]}(P, Q)}{\partial n} T(Q)\right] \mathrm{d} \Gamma(Q) \cdots
\end{gathered}
$$

ただし, 滑らかな境界上では $C=0.5$, 領域内部では $C=1$ であり, $\Gamma$ および $\Omega$ は境界および領域を示す. なお， $P$ および $Q$ は境界上の観測点とソース点であ り, 内点では $p, q$ を使用する. 二次元問題の場合, 式 (1) における定常温度解析の基本解 $T^{[1]}(P, Q)$ は次 式で与えられる.

$$
T^{[1]}(P, Q)=\frac{1}{2 \pi}\left[\ln \left(\frac{1}{r}\right)+C_{q}\right]
$$

$r$ は 2 点 $P, Q$ 間の距離であり, $C_{q}$ は, 任意の定数で ある、また, 上添字 $[1]$ は, 後で使用する $f$ 重多重調 和関数に扔いて $f=1$ であることを示す.

$2 \cdot 2$ 熱弾塑性解析 変形量があまり大きくない 場合の熱弾塑性問題は初期ひずみを伴う線形弾性問題 を繰返し解くことにより解を求めることができる。境

* 原稿受付 2008 年 1 月 23 日.

*1 正員, 近畿大学 (严 577-8502 東大阪市小若江 3-4-1).

E-mail : ochiai@mech.kindai.ac.jp 
界要素法を用いて任意の初期ひずみの増分 $\dot{\varepsilon}_{[j k}^{[1]}$ が作 用する場合, 次の境界積分方程式を解かなければなら ない(1)(2).

$$
\begin{aligned}
& c_{i j} \dot{u}_{j}(P)=\int_{\Gamma}\left[u_{i j}^{[1]}(P, Q) \dot{p}_{j}(Q)\right. \\
& \left.\quad-p_{i j}(P, Q) \dot{u}_{j}(Q)\right] \mathrm{d} \Gamma \\
& \quad+\int_{\Gamma}\left[\frac{\partial u_{i}^{T}(P, Q)}{\partial n} \dot{T}(Q)-u_{i}^{T}(P, Q) \frac{\partial \dot{T}(Q)}{\partial n}\right] \mathrm{d} \Gamma \\
& \quad+\int_{\Omega} \sigma_{i j k}^{[1]}(P, q) \dot{\varepsilon}_{i j k}^{[1]}(q) \mathrm{d} \Omega \cdots \cdots \cdots \cdots \cdots \cdots(3)
\end{aligned}
$$

ただし, 記号 $\dot{u}_{j}$ および $\dot{p}_{j}$ は変位増分および表面力増 分の $j$ 方向成分であり, $\Gamma$ および $\Omega$ は境界および領 域を示す.ディラックのデルタ関数を $\delta_{i j}$ とすると, $c_{i j}$ は滑らかな境界上では $0.5 \delta_{i j}$, 内点では $\delta_{i j}$ であ り, $\dot{T}$ は温度増分である. 式 ( 3 )に示すように初期ひ ずみが存在する場合, 領域積分を考慮しなければなら ない.ケルビンの解 $u_{i j}^{[1]}$ は二次元の場合

$$
\begin{array}{r}
u_{i j}^{[1]}=\frac{1}{8 \pi(1-\nu) G}\left[(3-4 \nu) \delta_{i j} \ln \left(\frac{1}{r}\right)+r_{, i} r_{, j}\right] \\
\cdots \cdots \cdots \cdots \cdots( \\
p_{i j}=\frac{-1}{4 \pi(1-\nu) r}\left\{\left[(1-2 \nu) \delta_{i j}+2 r_{, i} r_{, j}\right] \frac{\partial r}{\partial n}\right. \\
\left.-(1-2 \nu)\left(r_{, i} n_{j}-r_{, j} n_{i}\right)\right\} \cdots \cdots \cdots \cdots \cdots \cdots \cdots(
\end{array}
$$

で与えられる。ただし，rの $i$ 方向偏微分を $r_{, i}$, ポア ソン比を $\nu$, 線膨張係数を $\alpha$, 横弾性係数を $G$ とし, $n_{i}$ を単位法線ベクトルの $i$ 方向成分とする. 式 ( 3 ) における関数 $\sigma_{i j k}^{[1]}$ は, 次式で与えられる.

$$
\begin{aligned}
& \sigma_{i j k}^{[1]}=\left[(1-2 \nu)\left(\sigma_{i j} r_{, k}+\delta_{i k} r_{, j}-\delta_{j k} r_{, i}\right)\right. \\
& \left.\quad+2 r_{, i} r_{, j} r_{, k}\right] \frac{-1}{4 \pi(1-\nu) r} \cdots \cdots \cdots \ldots \ldots \ldots . . .
\end{aligned}
$$

初期ひずみ法において平面ひずみ問題の場合, 次の付 加項 $\sigma_{i j k}^{A[1]}$ が必要である.

$$
\sigma_{i j k}^{A[1]}=\frac{\nu r_{i} \delta_{j k}}{2 \pi(1-\nu) r}
$$

熱応力に関する関数 $u_{i}^{T}$ および $\partial u_{i}^{T} / \partial n$ は次式で与え られる(3).

$$
\begin{aligned}
& u_{i}^{T}=\frac{-m_{0} r r, i}{4 \pi}\left[\ln (r)-\frac{1}{2}\right] \ldots \ldots \ldots \ldots \ldots . . . \\
& \frac{\partial u_{i}^{T}}{\partial n}=\frac{m_{0}}{4 \pi}\left\{\left[\ln \left(\frac{1}{r}\right)-\frac{1}{2}\right] n_{i}-r_{, i} \frac{\partial r}{\partial n}\right\}
\end{aligned}
$$

ただし, 線熱膨張係数を $\alpha$ とすると平面応力問題では $m_{0}=(1+\nu) \alpha$, 平面ひずみ問題では $m_{0}=(1+\nu)$ $\alpha /(1-\nu)$ である.

初期応力法では, 初期応力増分を $\dot{\sigma}\left\{\begin{array}{l}11 \\ j k\end{array}(q)\right.$ とすると 式 ( 3 )の領域積分 П は次式となる.

$$
\Pi=\int_{\Omega} \varepsilon_{i j k}^{[1]}(P, q) \dot{\sigma}[1](q) \mathrm{d} \Omega
$$

ただし，

$$
\begin{aligned}
\varepsilon_{i j k}^{[1]} & =\left[(1-2 \nu)\left(\delta_{i j} r_{, k}+\delta_{i k} r_{, j}\right)-\delta_{j k} r_{, i}\right. \\
& \left.+2 r_{, i} r_{, j} r_{, k}\right] \frac{-1}{8 \pi(1-\nu) G r} \cdots \cdots \cdots . . .
\end{aligned}
$$

である. 式 ( 3 )，（10）には領域積分が含まれる.そこ で, 積分方程式を用いた補間法を活用して領域積分を 境界積分に変換する。

$2 \cdot 3$ 初期ひずみ分布の補間初期ひずみ $\dot{\varepsilon}[1] k$ ( $(q)$ はディラックのデルタ関数を用いて, 次式で表現する ことができる.

$$
\begin{aligned}
& \nabla^{2} \dot{\varepsilon}_{l j k}^{[1] S}(q)=-\dot{\varepsilon}_{j j k}^{[2] S}(q) \ldots \ldots \ldots \ldots . . . \\
& \nabla^{2} \dot{\varepsilon}_{j j k}^{[2] S}(q)=-\sum_{m=1}^{M} \dot{\varepsilon}_{j j k}^{[3] P} \delta\left(q-q_{m}\right)
\end{aligned}
$$

式(12)，(13)より, 次式が求められる.

$$
\nabla^{4} \dot{\varepsilon}_{l j k}^{[1] S}=\sum_{m=1}^{M} \dot{\varepsilon}_{l j k}^{[3 \mid P} \delta\left(q-q_{m}\right)
$$

式 (14) は, 境界積分方程式による自由曲面創成法に用 いられる式と同じものであり，2.5 次元の自由曲面を 示す式である.つまり, 本論文では初期ひずみ分布を 2.5 次元の自由曲面とみなしている。ただし, 補間は 初期ひずみ $\dot{\varepsilon}_{1 j k}^{[1]}(j, k=x, y)$ のそれぞれの成分に対し て考えるものとする(4)(5).

$2 \cdot 4$ 初期ひずみの積分表示 初期ひずみ分布の 境界積分方程式による表現方法に必要な $f$ 重調和関 数 $T^{[f]}$ は一般に次式で与えられる(6) (8).

$$
T^{[f]}=\frac{r^{2(f-1)}}{2 \pi[(2 f-2) ! !]^{2}}\left[\ln \left(\frac{1}{r}\right)+C_{q}+\operatorname{sgn}(f-1) \sum_{e=1}^{f-1} \frac{1}{e}\right]
$$

ただし,$(2 f) ! !=2 f(2 f-2) \cdots 4 \cdot 2$ である. 初期ひずみ増分 $\dot{\varepsilon}[1 j k$ は, 式(12)，(13)よりグリーンの定理より次式で与 えられる.

$$
\begin{aligned}
& c \dot{\varepsilon}_{l j k}^{[1] S}(P)=-\sum_{f=1}^{2}(-1)^{f} \int_{\Gamma}\left[T^{[f]}(P, Q) \frac{\dot{\varepsilon}_{\{j k}^{[f] S}(Q)}{\partial n}-\frac{\partial T^{[f]}(P, Q)}{\partial n} \dot{\varepsilon}_{Y j k}^{[f] S}(Q)\right] \mathrm{d} \Gamma-\sum_{m=1}^{M} T^{[2]}(P, q) \dot{\varepsilon}_{l j k(m)}^{[3] P}(q) \\
& C \dot{\varepsilon}_{[j k}^{[2] S}(P)=\int_{\Gamma}\left[T^{[1]}(P, Q) \frac{\partial \dot{\varepsilon}_{l j k}^{[2] S}(Q)}{\partial n}-\frac{\partial T^{[1]}(P, Q)}{\partial n} \dot{\varepsilon}_{[j k}^{[2] S}(Q)\right] \mathrm{d} \Gamma+\sum_{m=1}^{M} T^{[1]}(P, q) \dot{\varepsilon}_{[j j k(m)}^{[3] P}(q)
\end{aligned}
$$

連立積分方程式(16)，(17)を解くことにより補間を行う.

$2 \cdot 5$ 三重相反法 関数 $\sigma_{i j k}^{[1]}$ に対して次式を満足する関数 $\sigma_{i j k}^{[f]}$ が存在すると仮定する. 
$\nabla^{2} \sigma_{i j k}^{[f+1]}=\sigma_{i j k}^{[f]}$

式(12)，(13)，(18)およびグリーンの定理より，式（３）は次式になる.

$$
\begin{aligned}
& c_{i j} \dot{u}_{j}(P)=\int_{\Gamma}\left[u_{i j}^{[1]}(P, Q) \dot{p}_{j}(Q)-p_{i j}(P, Q) \dot{u}_{j}(Q)\right] \mathrm{d} \Gamma+\int_{\Gamma}\left[\frac{\partial u_{i}^{T}(P, Q)}{\partial n} \dot{T}(Q)-u_{i}^{T}(P, Q) \frac{\partial \dot{T}(Q)}{\partial n}\right] \mathrm{d} \Gamma \\
& +\sum_{f=1}^{2}(-1)^{f} \int_{\Gamma}\left[\frac{\partial \sigma_{i j k}^{[f+1]}(P, Q)}{\partial n} \dot{\varepsilon}_{i j k}^{[f] S}(Q)-\sigma^{[f+1]}{ }_{i j k}(P, Q) \frac{\partial \dot{\varepsilon}_{i j k}^{[j] S}(Q)}{\partial n}\right] \mathrm{d} \Gamma-\sum_{m=1}^{M} \sigma_{i j k}^{[3]}(P, q) \dot{\varepsilon}_{j j k(m)}^{[3] P}(q) \cdots
\end{aligned}
$$

同様に, 初期応力法では式 (10) は次式となる.

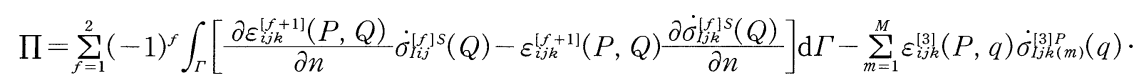

本手法では, 実際の計算において $\sigma_{i j k}^{[1]}$ は用いない. また, 次式で得られる関数 $u_{i j}^{[f]}$ を高次のケルビンの解と呼ぶこ とにする(4)(5).

$$
u_{i j}^{[f]}=\frac{-1}{2(1-\nu) G} T_{, i j}^{[f+1]}+\frac{\delta_{i j} T^{[f+1]}{ }_{k k}}{G}
$$

式(15)を式(21)に代入すると, 次式が得られる.

$$
u_{i j}^{[f]}=\frac{r^{2(f-1)}}{4 \pi[(2 f) ! !]^{2}(1-\nu) G}\left[\left[4 f(f-1) F_{2}+2(2 f-1)\right] r_{, i} \gamma_{, j}+\left\{\left[2 f(1-4 f)+8 f^{2} \nu\right] F_{2}+1-8 f(\nu-1)\right\} \delta_{i j}\right]
$$

ただし，

$$
\begin{aligned}
& F_{2}=\ln (r)-C_{q}-\sum_{e=2}^{f} \frac{1}{e} \\
& C_{q}=\frac{7-8 \nu}{2(3-4 \nu)}
\end{aligned}
$$

式(24)の条件を用いると, $f=1$ のとき従来のケルビン解 $(4)$ と同じになる. 式(22) から変位と応力の関係式扔よ び変位とひずみの関係式より高次基本解が得られる.

$$
\begin{aligned}
\sigma_{i j} & =\frac{2 \nu G}{(1-2 \nu)} \delta_{i j} u_{k, k}+G\left(u_{i, j}+u_{j, i}\right) \\
\varepsilon_{i j} & =\frac{1}{2}\left(u_{i, j}+u_{j, i}\right) \ldots \ldots \ldots \ldots \ldots \ldots \ldots \ldots \ldots \ldots \ldots \ldots \ldots \ldots \ldots \ldots
\end{aligned}
$$

式(22)を式(25)に代入すると $\sigma_{i j k}^{[f]}$ が得られる.

$$
\begin{aligned}
& \sigma_{i j k}^{[f]}=\frac{-2 r^{2 f-3}}{\pi(1-\nu)[(2 f) ! ! !]^{2}}\left[\left[-2 f(f-1)(f-2) F_{2}-\left(3 f^{2}-6 f+2\right)\right] r_{, i} r_{, j} \gamma_{, k}\right. \\
& +\left\{-2 f(f-1)\left(1-2 f_{\nu}\right) F_{2}-[1-2 f+2 f(3 f-2) \nu]\right\} \delta_{j k} r_{, i}+\left\{2 f\left[\left(2 f^{2}-3 f+1\right)-2 f(f-1) \nu\right] F_{2}\right. \\
& \left.\left.+\left(6 f^{2}-6 f+1\right)-2 f(3 f-2) \nu\right\}\left(\delta_{i j} r_{, k}+\delta_{k i} r_{, j}\right)\right]
\end{aligned}
$$

式(27)の法線方向微分は, 次式となる.

$$
\begin{aligned}
& \frac{\partial \sigma_{i j k}^{[f]}}{\partial n}=\frac{-r^{2(f-2)}}{\pi(1-\nu)[(2 f) ! !]^{2}}\left[\left\{2 f(f-1)[1-2 f(1-\nu)] F_{2}+6 f^{2}(1-\nu)-2 f(3-2 \nu)+1\right\}\left(\sigma_{i k} n_{j}+\delta_{j i} n_{k}\right)\right. \\
& -2\left\{2 f(f-1)(f-2)[1-2 f(1-\nu)] F_{2}+\left(-8 f^{3}+21 f^{2}-14 f+2\right)+2 f\left(4 f^{2}-9 f+4\right) \nu\right\}\left(\delta_{i k} r_{j}+\delta_{j i} r_{, k}\right) r_{, s} n_{s} \\
& +\left\{2 f(f-1)(2 \nu f-1) F_{2}+\left[1+6 \nu f^{2}-2 f(1+2 \nu)\right]\right\} \delta_{j k} n_{j}+2\left[2 f(f-1)(f-2)(2 \nu f-1) F_{2}\right. \\
& \left.-\left(3 f^{2}-6 f+2\right)+2 f\left(4 f^{2}-9 f+4\right) \nu\right] \delta_{j k} r_{, i} r_{, s} n_{s}+8\left[-f(f-1)(f-2)(f-3) F_{2}\right. \\
& \left.-\left(2 f^{3}+9 f^{2}-11 f+3\right)\right] r_{, i} r_{, j} r_{, k} r_{,} n_{s}+2\left[-2 f(f-1)(f-2) F_{2}\right. \\
& \left.\left.-\left(3 f^{2}-6 f+2\right)\right]\left(n_{i} r_{j} r_{, k}+n_{j} r_{, i} r_{, k}+n_{k} r_{, i} r_{, j}\right)\right] \cdots
\end{aligned}
$$

付加項の高次基本解およびその法線微分係数は, 同様に次式で与えられる(4).

$$
\begin{aligned}
& \sigma_{i j k}^{A[f]}=\frac{\nu \delta_{j k} r_{i} \gamma^{2 f}}{2(1-\nu) \pi[(2 f-2) ! !]^{2}}\left[2(f-1) F_{1}+1\right] \\
& \frac{\partial \sigma_{i j k}^{A f f]}}{\partial n}=\frac{\nu \delta_{j k} r^{2 f-1}}{2(1-\nu) \pi[(2 f-2) ! !]^{2}}\left\{\left[n_{i}+2(f-2) r_{, i} r_{s} n_{s}\right]\left[2(f-1) F_{1}+1\right]+2(f-1) r_{, i} r_{, s} n_{s}\right\} \\
& F_{1}=\ln (r)-C_{q}-\sum_{e=1}^{f-1} \frac{1}{e}
\end{aligned}
$$

初期応力法に必要な関数 $\varepsilon_{i j k}^{[f]}$ は, 式(22)を式(26)に代入すると得られる. 


$$
\begin{aligned}
\varepsilon_{i j k}^{[f]} & =\frac{r^{2 f-3}}{2 \pi G[(2 f) ! !]^{2}}\left\{\frac{2}{(1-\nu)}\left[2 f(f-1)(f-2) F_{2}+\left(3 f^{2}-6 f+2\right)\right] r_{, i} r_{, j} r_{, k}\right. \\
& \left.+\left[2 f(f-1) F_{2}+(2 f-1)\right]\left(\delta_{i k} r_{, j}+\delta_{j k} r_{, i}+\delta_{i j} r_{, k}\right)+2\left[2 f^{2}(f-1) F_{2}+f(3 f-2)\right]\left(\delta_{i j} r_{, k}+\delta_{i k} r_{, j}\right)\right\}
\end{aligned}
$$

式(32)の法線方向微分係数は次式となる.

$$
\begin{aligned}
& \frac{\partial \varepsilon_{i j k}^{[f]}}{\partial n}=\frac{r^{2 f-4}}{2 \pi G(1-\nu)[(2 f) ! !]^{2}}\left[8\left\{(2 f-3)[1+f(f-3)]+f(f-1)(f-2)(f-3) F_{2}\right\} r_{, i} r_{, j} r_{, k} r_{, s} n_{s}\right. \\
& +2\left[3 f^{2}-6 f+2+2 f(f-1)(f-2) F_{2}\right]\left(r_{, j} r_{, k} n_{i}+r_{, i} r_{, k} n_{j}+r_{, i} r_{, j} n_{k}+\delta_{j k} r_{, i} r_{, s} n_{s}\right) \\
& +2\left\{2-2 f(7-4 \nu)-8 f^{3}(1-\nu)+3 f^{2}(7-6 \nu)+2 f(f-1)(f-2)[-2 f(1-\nu)+1] F_{2}\right\}\left(\delta_{i j} r_{, k}+\delta_{i k} r_{, j}\right) r_{, s} n_{s} \\
& -\left[-2 f(f-1) F_{2}-2 f+1\right] \delta_{j k} n_{i}-\left\{1+6 f^{2}(1-\nu)-2 f(3-2 \nu)\right. \\
& \left.\left.-2 f(f-1)[-2 f(1-\nu)+1] F_{2}\right\}\left(\delta_{i j} n_{k}+\delta_{i k} n_{j}\right)\right]
\end{aligned}
$$

$2 \cdot 6$ 内部応力 一般に内部応力は次式で与えられる.

$$
\begin{aligned}
& \dot{\sigma}_{i j}(p)=\int_{\Gamma}\left[-\sigma_{k i j}^{[1]}(p, Q) \dot{p}_{k}(Q)-S_{k i j}(p, Q) \dot{u}_{k}(Q)\right] \mathrm{d} \Gamma+\int_{\Gamma}\left[\frac{\partial \sigma_{i j}^{T}(p, Q)}{\partial n} \dot{T}(Q)-\sigma_{i j}^{T}(p, Q) \frac{\partial \dot{T}(Q)}{\partial n}\right] \mathrm{d} \Gamma \\
& +\int_{\Omega} \sigma_{i j k s}^{[1]}(p, q) \dot{\varepsilon}_{l k s}^{[1]}(q) \mathrm{d} \Omega-\dot{\sigma}_{i j}^{11}(p)
\end{aligned}
$$

式(34)の $\left.\dot{\sigma}\right|_{i j} ^{[1]}$ は $\dot{\varepsilon}_{Y i j}^{[1]}$ を, 次式に代入することにより求められる初期応力成分である.

$$
\dot{\sigma}[i]=2 G \dot{\varepsilon}[1]+\lambda \dot{\varepsilon}_{l k k}^{[1]} \delta_{i j}
$$

ただし, $\lambda$ はラメの常数である。また, 式(34)の $S_{k i j}, \sigma_{i j k s}^{[1]}$ および $\sigma_{i j}^{T}, \partial \sigma_{i j}^{T} / \partial n$ は次式で与えられる.

$$
\begin{aligned}
& S_{k i j}=\frac{2 G}{r^{2}}\left\{2 \frac{\partial r}{\partial n}\left[(1-2 \nu) \delta_{i j} r_{, k}+\nu\left(\delta_{i k} r_{, j}+\delta_{j k} r_{, i}\right)-4 r_{, i} r_{, j} r_{, k}\right]+2 \nu\left(n_{i} r_{, j} r_{, k}+n_{j} r_{, i} r_{, k}\right)\right. \\
& \left.+(1-2 \nu)\left(2 n_{k} r_{, i} r_{, j}+n_{j} \delta_{i k}+n_{i} \delta_{j k}\right)-(1-4 \nu) n_{k} \delta_{i j}\right\} \frac{1}{4 \pi(1-\nu)} \\
& \sigma_{i j k s}^{[1]}=\frac{1}{2 \pi(1-\nu) r^{2}}\left[2(1-2 \nu)\left(\delta_{i j} r_{, k} r_{, s}+\delta_{k s} \gamma_{, i} r_{, j}\right)+2 \nu\left(\delta_{s i} r_{, k} r_{, j}+\delta_{j k} r_{, i} r_{, s}+\delta_{i k} r_{, s} r_{, j}+\delta_{j s} r_{, k} r_{, i}\right)\right. \\
& \left.-8 r_{, i} r_{, j} r_{, k} r_{, s}+(1-2 \nu)\left(\delta_{i k} \delta_{s j}+\delta_{j k} \delta_{s i}\right)-(1-4 \nu) \delta_{i j} \delta_{k s}\right] \\
& \sigma_{i j}^{T}=\frac{G m_{0}}{2 \pi r}\left\{r_{, i} r_{, j}+\delta_{i j}\left[-\frac{1}{2}+\ln \left(\frac{1}{r}\right)\right]\right\} \\
& \frac{\partial \sigma_{i j}^{T}}{\partial n}=\frac{G m_{0}}{2 \pi r}\left[-\frac{\partial r}{\partial n}\left(\sigma_{i j}+2 r_{, i} r_{, j}\right)+n_{i} r_{, j}+n_{j} r_{, i}\right]
\end{aligned}
$$

関数 $\sigma_{i j k s}^{[1]}$ に対して次式を満足する関数 $\sigma_{i j k s}^{[f]}$ が存在すると仮定する.

$\nabla^{2} \sigma_{i j k s}^{[f+1]}=\sigma_{i j k s}^{[f]}$

式(34)は式(40)より次式となる.

$$
\begin{aligned}
& \dot{\sigma}_{i j}(p)=\int_{\Gamma}\left[-\sigma_{k i j}^{[1]}(p, Q) \dot{p}_{k}(Q)-S_{k i j} \dot{u}_{k}(Q)\right] \mathrm{d} \Gamma+\int_{\Gamma}\left[\frac{\partial \sigma_{i j}^{T}(p, Q)}{\partial n} \dot{T}(Q)-\sigma_{i j}^{T} \frac{\partial \dot{T}(Q)}{\partial n}\right] \mathrm{d} \Gamma \\
& \quad+\sum_{f=1}^{2}(-1)^{f} \int_{\Gamma}\left[\frac{\partial \sigma_{i j k s}^{[f]}(p, Q)}{\partial n} \dot{\varepsilon}_{l k S}^{[f] S}(Q)-\sigma_{i j k s}^{[f]}(p, Q) \frac{\partial \dot{\varepsilon}(f) S(Q)}{\partial n}\right] \mathrm{d} \Gamma-\sum_{m=1}^{M} \sigma_{i j k s}^{[3]}(p, Q) \dot{\varepsilon}_{k s(m)}^{[3] P}(q)-\dot{\sigma}_{I i j}^{[1]}(p)
\end{aligned}
$$

式(27)および変位と応力の関係式 $(25) よ り ， \sigma_{i j k s}^{[f]}$ は次式で与えられる.

$$
\begin{aligned}
& \sigma_{i j k s}^{[f]}=\frac{-2 r^{2(f-2)} G}{\pi[(2 f) ! !]^{2}(1-\nu)}\left[2\left[2 f(f-1)(f-2)(2 \nu f-1) F_{2}+2 \nu f\left(4 f^{2}-9 f+4\right)-\left(3 f^{2}-6 f+2\right)\right] \delta_{i s} \gamma_{, j} \gamma_{, k}\right. \\
& +\frac{2 \nu f}{1-2 \nu}\left\{2 f(f-1)[2 \nu(f-2)+1] F_{2}+(3 f-2)+2\left(4 f^{2}-9 f+4\right) \nu\right\} \delta_{i s} \delta_{j k} \\
& -8\left[f(f-1)(f-2)(f-3) F_{2}+\left(2 f^{3}-9 f^{2}+11 f-3\right)\right] r_{, i} r_{, j} r_{, k} r_{, s}+\left\{-2 f(f-1)[1-2 f(1-\nu)] F_{2}\right. \\
& \left.+6 f^{2}(1-\nu)-2 f(3-2 \nu)+1\right\}\left(\delta_{s k} \delta_{i j}+\delta_{j s} \delta_{k i}\right)+\left[2 f(f-1)(2 \nu f-1) F_{2}+6 \nu f^{2}-2 f(1+2 \nu)+1\right] \delta_{j k} \delta_{s i} \\
& +2\left[2 f(f-1)(f-2)(2 \nu f-1) F_{2}+\left(-3 f^{2}+6 f-2\right)+2 f\left(4 f^{2}-9 f+4\right) \nu\right] \delta_{j k} r_{, s} r_{, i} \\
& +2\left\{-2 f(f-1)(f-2)[1-f(1-\nu)] F_{2}+2\left(2 f^{3}-6 f^{2}+5 f-1\right)\right. \\
& \left.\left.-\nu f\left(4 f^{2}-9 f+4\right)\right\}\left(\delta_{j s} r_{, k} r_{, i}+\delta_{k s} r_{, i} r_{, j}+\delta_{j i} r_{, k} r_{, s}+\delta_{k i} r_{, s} r_{, j}\right)\right]
\end{aligned}
$$

また, 本論文に必要な式(42)の法線方向の微分は次式で与えられる. 


$$
\begin{aligned}
& \frac{\partial \sigma_{i j k s}^{[2]}}{\partial n}=\frac{G}{32(1-\nu) \pi r}\left\{\frac{4 \nu \delta_{i j} \delta_{k s} r_{, m} n_{m}}{(1-2 \nu)}+\left[(3-4 \nu)\left(\delta_{i s} \delta_{j k}+\delta_{j s} \delta_{i k}\right)-(1-4 \nu) \delta_{i j} \delta_{k s}\right)\right] r_{, m} n_{m} \\
& +(1-2 \nu)\left[\delta_{j s}\left(n_{i} r_{, k}+n_{k} r_{, i}-2 r_{, k} r_{, i} r_{, m} n_{m}\right)+\delta_{i k}\left(n_{j} r_{, k}+n_{k} r_{, j}-2 r_{, k} r_{, j} r_{, m} n_{m}\right)\right. \\
& \left.+\delta_{j k}\left(n_{i} r_{, k}+n_{k} r_{, i}-2 r_{, k} r_{, i} r_{, m} n_{m}\right)+\delta_{k s}\left(n_{j} r_{, i}+n_{i} r_{, j}-2 r_{, i} r_{, j} r_{, m} n_{m}\right)\right] \\
& \left.+2\left(n_{i} r_{, j} r_{, k} r_{, s}+n_{j} r_{, i} r_{, k} r_{, s}+n_{k} r_{, j} r_{, i} r_{, s}+n_{s} r_{, i} r_{, j} r_{, k}-4 r_{, i} r_{, i} r_{, k} r_{, s} r_{, m} n_{m}\right)\right\} \cdots \\
& \frac{\partial \sigma_{i j k s}^{[3]}}{\partial n}=\frac{-G r}{756(1-\nu) \pi}\left[\frac{36 \nu \delta_{s k} \delta_{i j} r_{, m} n_{m}(1+2 \nu)}{(1-2 \nu)}\left\{2\left[\ln (r)-C_{q}\right]+1\right\}-12(1-6 \nu)\left(\delta_{i j} r_{, s} r_{, k}+\delta_{s k} r_{, i} r_{, j}\right) r_{, m} n_{m}\right. \\
& +12(2-3 \nu)\left(\delta_{i s} r_{, j} r_{, k}+\delta_{j s} r_{, i} r_{, k}+\delta_{i k} r_{, j} r_{, s}+\delta_{j k} r_{, i} r_{, s}\right)+24 r_{, k} r_{, i} r_{, s} r_{, m} n_{m} \\
& +\delta_{i j} \delta_{k s} r_{, m} n_{m}\left\{-12(1-6 \nu)\left[\ln (r)-C_{q}\right]-1\right\}+r_{, m} n_{m}\left(\delta_{i s} \delta_{k j}+\delta_{j s} \delta_{k i}\right)\left[12(5-6 \nu)+\ln (r)-C_{q}+(17-18 \nu)\right] \\
& +\left[\delta_{i j}\left(n_{s} r_{, k}+n_{k} r_{, s}\right)+\delta_{s k}\left(n_{i} r_{, j}+n_{j} r_{, i}\right)\right]\left\{-12(1-6 \nu)\left[\ln (r)-C_{q}\right]-1+18 \nu\right\} \\
& +\left[\delta_{i s}\left(n_{j} r_{, k}+n_{k} r_{, j}\right)+\delta_{j s}\left(n_{i} r_{, k}+n_{k} r_{, i}\right)+\delta_{i k}\left(n_{j} r_{, s}+n_{s} r_{, j}\right) \delta_{j k}\left(n_{i} r_{, s}+n_{s} r_{, i}\right)\right] \\
& \left.\times\left\{12(2-3 \nu)\left[\ln (r)-C_{q}\right]+8-9 \nu\right\}-12\left(n_{k} r_{, i} r_{, j} r_{, s}+n_{i} r_{, k} r_{, j} r_{, s}+n_{i} r_{, i} r_{, k} r_{, s}+n_{s} r_{, i} r_{, j} r_{, k}\right)\right]
\end{aligned}
$$

付加項の高次基本解およびその法線微分係数は次式で与えられる.

$$
\begin{aligned}
& \sigma_{i j k s}^{A[f]}=\frac{\nu G \delta_{k s} \gamma^{2 f-4}}{(1-\nu)(1-2 \nu)[(2 f-2) ! !]^{2} \pi}\left[4 \nu \delta_{i j}(f-1)\left[(f-1) F_{1}+1\right]\right. \\
& \left.+(1-2 \nu)\left\{\left[\delta_{i j}+2(f-2) r_{, i} r_{, j}\right]\left[2(f-1) F_{1}+1\right]+2(f-1) r_{, i} r_{, j}\right\}\right] \\
& \frac{\partial \sigma_{i j k s}^{A[f]}}{\partial n}=\frac{2 \nu G \delta_{k s} \gamma^{2 f-5}}{(1-\nu)(1-2 \nu)[(2 f-2) ! !]^{2} \pi}\left[4(f-1)(f-2) \nu \delta_{i j} \gamma_{, m} n_{m}\left[(f-1) F_{1}+1\right]+(f-2)(1-2 \nu)\right. \\
& \times\left\{\left[\delta_{i j} r_{, m} n_{m}+2(f-3) r_{, m} n_{m} r_{, i} r_{, j}+n_{i} r_{, j}+n_{j} r_{, i}\right]\left[2(f-1) F_{1}+1\right]+2(f-1) r_{, m} n_{m} r_{, i} r_{, j}\right\} \\
& \left.+2 \nu(f-1)^{2} \delta_{i j} r_{, m} n_{m}+(1-2 \nu)\left\{(f-1)\left[\delta_{k s}+2(f-3) r_{, i} r_{, j}\right] r_{, m} n_{m}+(f-1)\left(n_{i} r_{, j}+n_{j} r_{, i}\right)\right\}\right]
\end{aligned}
$$

初期応力関数に関する関数は, 式(32)を式(25)に代入することにより次式となる.

$$
\begin{aligned}
& \varepsilon_{i j k s}^{[f]}=\frac{r^{2 f-4}}{\pi(1-\nu)[(2 f) ! !]^{2}}\left[\left\{-2\left[8 f^{3} \nu-3 f^{2}(1+6 \nu)+2 f(3+4 \nu)-2\right]-4 f(f-1)(f-2)(2 f \nu-1) F_{2}\right\} \delta_{i s} r_{, j} r_{, k}\right. \\
& -\left[6 f^{2} \nu-2 f(1+2 \nu)+1+2 f(f-1)(2 f \nu-1) F_{2}\right] \delta_{i s} \delta_{j k}+\{8(2 f-3)[1+f(f-3)] \\
& \left.+f(f-1)(f-2)(f-3) F_{2}\right\} r_{, i} r_{, j} r_{, k} r_{, s}+2\left[3 f^{2}-6 f+2+2 f(f-1)(f-2) F_{2}\right] \delta_{j k} r_{, i} r_{, s} \\
& -2\left\{4 f^{3}(1-\nu)-3 f^{2}(4-3 \nu)+2 f(5-2 \nu)-2-2 f(f-1)(f-2)[1-f(1-\nu)] F_{2}\right\} \\
& \times\left(\delta_{i j} r_{, k} r_{, s}+\delta_{i k} r_{, j} r_{, s}+\delta_{s j} r_{, k} r_{, i}+\delta_{s k} r_{, j} r_{, i}\right)-\left\{1+6 f^{2}(1-\nu)-2 f(3-2 \nu)\right. \\
& \left.\left.+2 f(f-1)[2 f(1-\nu)-1] F_{2}\right\}\left(\delta_{i j} \delta_{k s}+\delta_{i k} \delta_{j s}\right)\right]
\end{aligned}
$$

また, 式(48)の法線方向の微分は次式で与えられる.

$$
\begin{aligned}
& \frac{\partial \varepsilon_{i j k s}^{[2]}}{\partial n}=\frac{1}{16(1-\nu) r \pi}\left\{( 1 - 2 \nu ) \left[\delta_{i k} r_{, j} n_{s}+\delta_{i k} r_{, s} n_{j}+\delta_{i s} r_{, j} n_{k}+\delta_{i s} r_{, k} n_{j}+\delta_{j k} r_{, i} n_{s}+\delta_{j k} r_{, s} n_{i}\right.\right. \\
& \left.+\delta_{j s} r_{, i} n_{k}+\delta_{j s} r_{, k} n_{i}-2\left(\delta_{i k} r_{, j} r_{, s}+\delta_{i s} r_{, j} r_{, k}+\delta_{j k} r_{, i} r_{, s}+\delta_{j s} r_{, i} r_{, k}\right) r_{, m} n_{m}\right] \\
& -(1-4 \nu)\left(\delta_{i j} \delta_{s k} r_{, m} n_{m}+\delta_{i j} r_{, s} n_{k}+\delta_{i j} r_{, k} n_{s}-2 \delta_{i j} r_{, k} r_{, s} r_{, m} n_{m}\right) \\
& +(3-4 \nu)\left(\delta_{i k} \delta_{j s}+\delta_{i s} \delta_{j k}\right) r_{, m} n_{m}-\delta_{k s} r_{, j} n_{i}-\delta_{k s} r_{, i} n_{j}+2 \delta_{k s} r_{, i} r_{, j} r_{, m} n_{m} \\
& \left.+2\left(r_{, i} r_{, j} r_{, s} n_{k}+r_{, i} r_{, j} r_{, k} n_{s}+r_{, j} r_{, k} r_{, s} n_{i}+r_{, i} r_{, k} r_{, s} n_{j}\right)-8 r_{, i} r_{, j} r_{, s} r_{, k} r_{, m} n_{m}\right\} \\
& \frac{\partial \varepsilon_{i j k s}^{[3]}}{\partial n}=\frac{r}{1152(1-\nu) \pi}\left[\{ 1 2 ( 2 - 3 \nu ) [ \operatorname { l n } ( r ) - C _ { q } ] + 8 - 9 \nu \} \left(\delta_{i k} r_{, s} n_{j}+\delta_{i k} r_{, j} n_{s}+\delta_{i s} r_{, k} n_{j}+\delta_{i s} r_{, j} n_{k}\right.\right. \\
& \left.+\delta_{j k} r_{, s} n_{i}+\delta_{j k} r_{, i} n_{s}+\delta_{j s} r_{, k} n_{i}+\delta_{j s} r_{, i} n_{k}\right)+\left\{12(5-6 \nu)\left[\ln (r)-C_{q}\right]+17-18 \nu\right\}\left(\delta_{i k} \delta_{j s}+\delta_{i s} \delta_{j k}\right) r_{, m} n_{m} \\
& -\left\{12(1-6 \nu)\left[\ln (r)-C_{q}\right]+1-18 \nu\right\}\left(\delta_{i j} \delta_{k s} r_{, m} n_{m}+\delta_{i j} r_{, s} n_{k}+\delta_{i j} r_{, k} n_{s}\right) \\
& +\left\{12\left[\ln (r)-C_{q}\right]+1\right\}\left(\delta_{k s} \gamma_{, i} n_{j}+\delta_{k s} \gamma_{, j} n, i\right)+12(2-3 \nu)\left(\delta_{i k} r_{, j} \gamma_{, s}+\delta_{i s} \gamma_{, j} \gamma_{, k}+\delta_{j k} r_{, i} \gamma_{, s}+\delta_{j s} \gamma_{, i} \gamma_{, k}\right) r_{, m} n_{m} \\
& -12(1-6 \nu) \delta_{i j} r_{, k} r_{, s} r_{, m} n_{m}-12\left(\delta_{k s} r_{, i} r_{, j} r_{, m} n_{m}+r_{, i} r_{, s} r_{, j} n_{k}+r_{, i} r_{k} r_{, j} n_{s}+r_{, k} r_{, s} r_{, j} n_{i}+r_{, i} r_{, k} r_{, s} n_{j}\right) \\
& \left.+24 r_{, i} r_{, j} r_{, k} r_{, s} r_{, m} n_{m}\right]
\end{aligned}
$$

繰返し計算により熱弾塑性解析を行う。まず, 温度分布および塑性が開始する温度 $T_{S}$ を求め, 最終温度を $T_{F}$, 時間分割数を $N$ とし, $\left(T_{F}-T_{S}\right) / N$ の温度を順次加えていく. 式 $(3)$ よ変位おょび反力を求め, 内部応力および 表面応力より次のテップの初期ひずみを求める. 初期ひずみの収束を判定した後, 次のステップ計算を行う. 


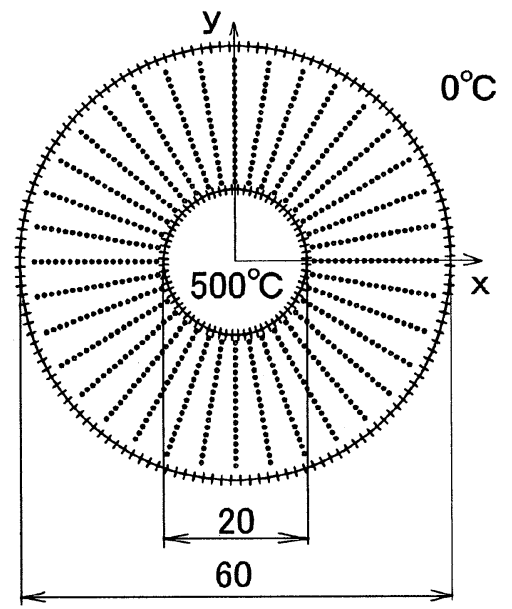

Fig. 1 Circular cylinder

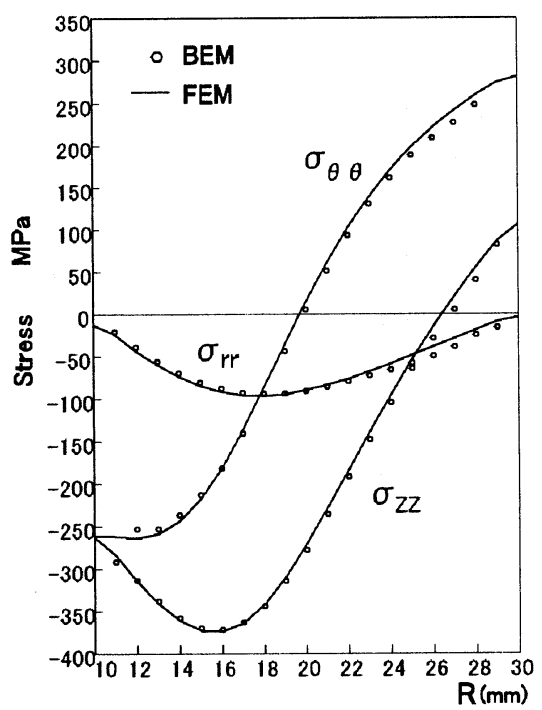

Fig. 2 Thermal stress distribution in circular cylinder

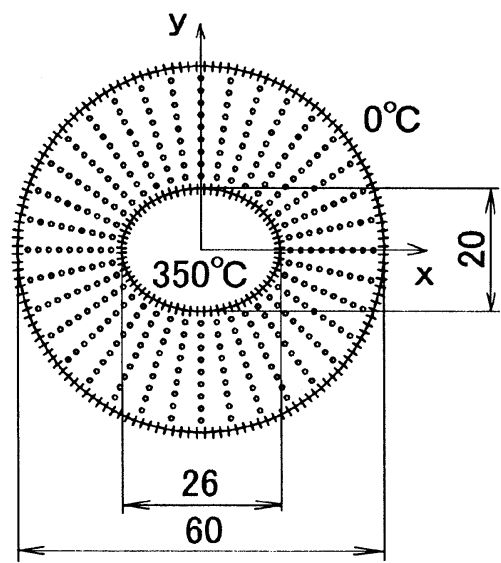

Fig. 3 Circular plate with elliptical hole

\section{3. 解 析 例}

内表面の温度が $500^{\circ} \mathrm{C}$, 外表面の温度が $0^{\circ} \mathrm{C}$ 円筒 の熱弾塑性解析を行った. $0^{\circ} \mathrm{C}$ 基準温度とし, 温度 分布は定常状態, 円筒は弾塑性体とし，ミーゼスの降 伏条件を使用し，平面ひずみ状態と仮定した。内径 20 $\mathrm{mm}$, 外径を $60 \mathrm{~mm}$ とし, 縦弾性係数 $E=210 \mathrm{GPa}$, ポアソン比 $\nu=0.3$, 降伏点応力を $Y=250 \mathrm{MPa}$ とし, 線膨張係数を $0.000011 \mathrm{~K}^{-1}$ とした. 加工硬化係数は $0.1 Y$ とした. 図 1 に示す内点を使用し, 本手法での 計算結果と有限要素による解との比較を図 2 に示す.

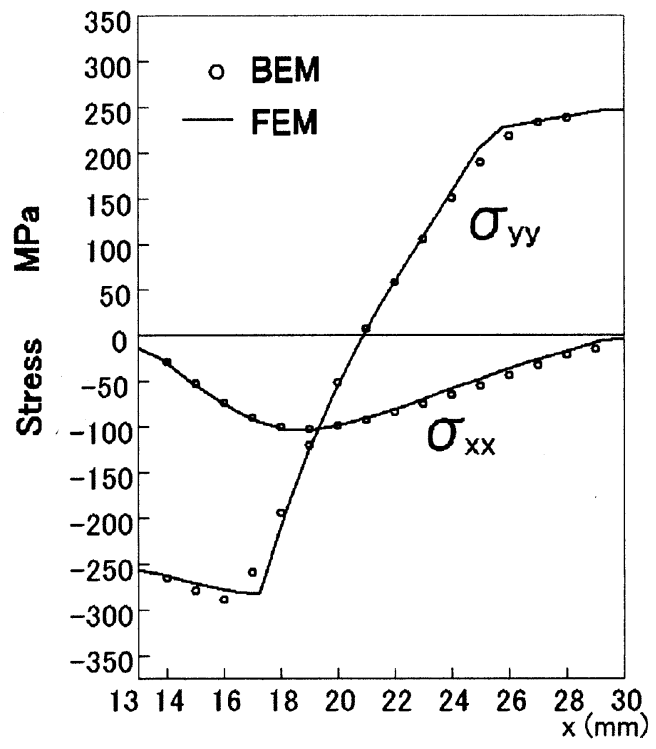

Fig. 4 Thermal stress distribution $(y=0 \mathrm{~mm})$

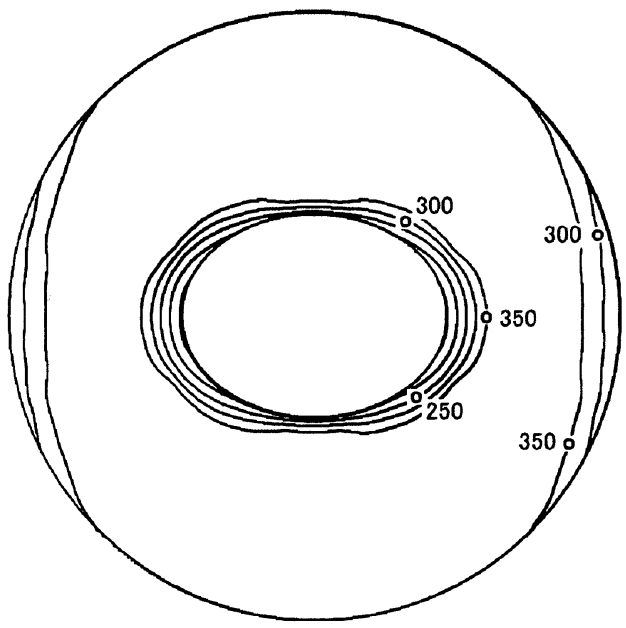

Fig. 5 Plastic zone for different temperature 
次に，だ円孔を有する弾完全塑性体の円板の熱弾性 解析を行った. 内表面の温度を $350^{\circ} \mathrm{C}$, 外表面の温度 を $0^{\circ} \mathrm{C} と し$, 温度分布は定常状態とした. 有限要素 法 ${ }^{(9)}$ による解析結果と精度の比較を行った。 だ円孔 の長径を $26 \mathrm{~mm}$, 短径を $20 \mathrm{~mm}$ とし, 縦弾性係数 $E$ $=210 \mathrm{GPa}$, ポアソン比 $\nu=0.3$, 降伏点応力を $Y=$ $250 \mathrm{MPa}$ とし, 線膨張係数を $0.000011 \mathrm{~K}^{-1}$ とした。 図 3 に示す内点を使用し, 本手法での計算結果と有限 要素法 ${ }^{(9)}$ での值との比較を図 4 に示す. 図 5 に内側 表面温度を変化させた場合の塑性領域の拡がりを示 す.

\section{4. 結言}

内部セルを用いていた熱弾塑性問題を, 内部セルを 用いないで解析が可能なことがわかった。これによ り, データの入力が容易である境界要素法の利点が, 維持できることが示された.なお, 必要となる高次基 本解を一般式で示した.

\section{文献}

(1) Brebbia, C. A., Boundary Element Techniques - Theory and Applications in Engineering, (1984), pp. 49-64, Springer-Verlag.
(2) Telles, J. F. C., The Boundary Element Method Applied to Inelastic Problems, (1983), pp. 42-45, Springer-Verlag.

(3) Sekiya, T., Ochiai, Y. and Ishida, R., Thermal Stress Analysis by Boundary Element Method (in Japanese), (1992), pp. 33-52, Morikita.

(4) Ochiai, Y. and Kobayashi, T., Initial Strain Formula tion without Internal Cells for Elastoplastic Analysis by Triple-Reciprocity BEM, International Journal for Numerical Methods in Engineering, Vol.50 (2001), pp. 1879-1891.

(5) Ochiai, Y. and Kobayashi, T., Initial Stress Formulation for Elastoplastic Analysis by Improved MultipleReciprocity Boundary Element Method, Engineering Analysis with Boundary Elements, Vol.23 (1999), pp. 167-173.

(6) Ochiai, Y., Multidimensional Numerical Integration for Meshless BEM, Engineering Analysis with Bound. ary Elements, Vol. 27, No. 3 (2003), pp. 241-249.

(7) Ochiai, Y. and Seto, S., Elastic Analysis with Centrifugal Load in Non-Homogeneous Materials by TripleReciprocity Boundary Element Method, Transaction of Japan Society of Mechanical Engineers, Vol.72, No. 722 (2006), pp. 1458-1463.

(8) Ochiai, Y. and Harada, K., Axial Symmetric Stationary Heat Conduction Analysis on Functional Gradient Materials by Triple-Reciprocity BEM, Transaction of Japan Society of Mechanical Engineers, Vol.73, No. 735 (2007), pp. 2290-2296.

(9) Nakasone, Y. et al., Engineering Analysis with ANSYS Software, (2007), pp. 263-325, Butterworth-Heineman. 